\title{
EVIDENCE FOR THE EXISTENCE \\ OF MASS-EXCHANGE BINARY Be STARS \\ FROM PERIODIC SPECTRAL VARIATIONS
}

\author{
GERALDINE J. PETERS \\ Dept. of Astronomy, University of California, Calif., Los Angeles, U.S.A.
}

\begin{abstract}
In this paper, we discuss the Be stars which show recurrent shell structure and/or cyclic variations in the profiles of their emission lines with periods near $100^{\mathrm{d}}$ and, thus, are good candidates for interacting binaries undergoing mass-exchange. The periodic spectral variations observed in the B1e star HR 2142 are reviewed and brought up to date in the light of recent observations. HR 2142 is then compared with other periodic binary Be stars (AX Mon, 17 Lep, HD 218393, HD 173219, and $\phi$ Per) in order to point out the wide variance in observed spectra which interacting binaries can display.
\end{abstract}

\section{Introduction}

The simplest way to explain the occurrence of periodic shell structure or cyclic variations in the profiles of emission lines in Be stars is in terms of binary motion of an interacting system. As the binary presents different aspects, the column density of absorbers and the projected envelope geometry vary as a result of an asymmetrical distribution of material caused by mass streaming. In this paper, we will be concerned with $\mathrm{Be}$ stars which show variations with periods around $100^{d}$ and, thus, can be considered as good candidates for interacting binary systems undergoing Case B mass exchange.

In connection with periodic activity, I would like to discuss HR 2142, a B1e star whose spectrum I have studied extensively for the past six years. HR 2142 (HD 41335, MWC 133, BD $-6^{\circ} 1391$ ) periodically displays a conspicuous, shorttermed, two-component shell phase every $80^{d} .86$. Outside of shell phase $(90 \%$ of the cycle), HR 2142 very much resembles what most researchers studying Be stars would call the prototype 'classical Be star'. The photospheric features are extremely broad while $\mathrm{H} \alpha-\mathrm{H} \delta$ contain conspicuous emission features superposed on their respective photospheric profiles. In addition, HR 2142 has emission features of $\mathrm{Fe}$ II, $\mathrm{O}_{\text {I }}$ $\lambda 7774 \AA$, and $O_{\text {I }} \lambda 8446 \AA$ which are sometimes observed in 'classical' Be stars. However, every $81^{\mathrm{d}}$ the sequence of events shown in Figure 1 occurs. The Balmer lines develop deep shell features which persist for about $5^{\mathrm{d}}$, disappear, then reappear again at the same strength for the short duration of 1.5 . The first segment of the shell phase is termed the primary shell phase while the second part is called the secondary shell phase. The details of the shell phase of HR 2142 are discussed below. In Section 3 we will present a model for HR 2142 based upon the observations. Finally, toward the end of the paper HR 2142 is compared with some well-studied binary Be stars.

The spectrograms used in the analysis of HR 2142 were obtained with the coude spectrograph of the 120 -in. $(305 \mathrm{~cm})$ telescope at Lick Observatory and with the cassegrain spectrograph of the UCLA 24 -in. $(61 \mathrm{~cm})$ telescope at Ojai, California. The Lick Observatory spectrograms were taken with both the 120-in. telescope and the 24-in. Coudé Auxiliary Telescope and include Varo image tube spectra in the 


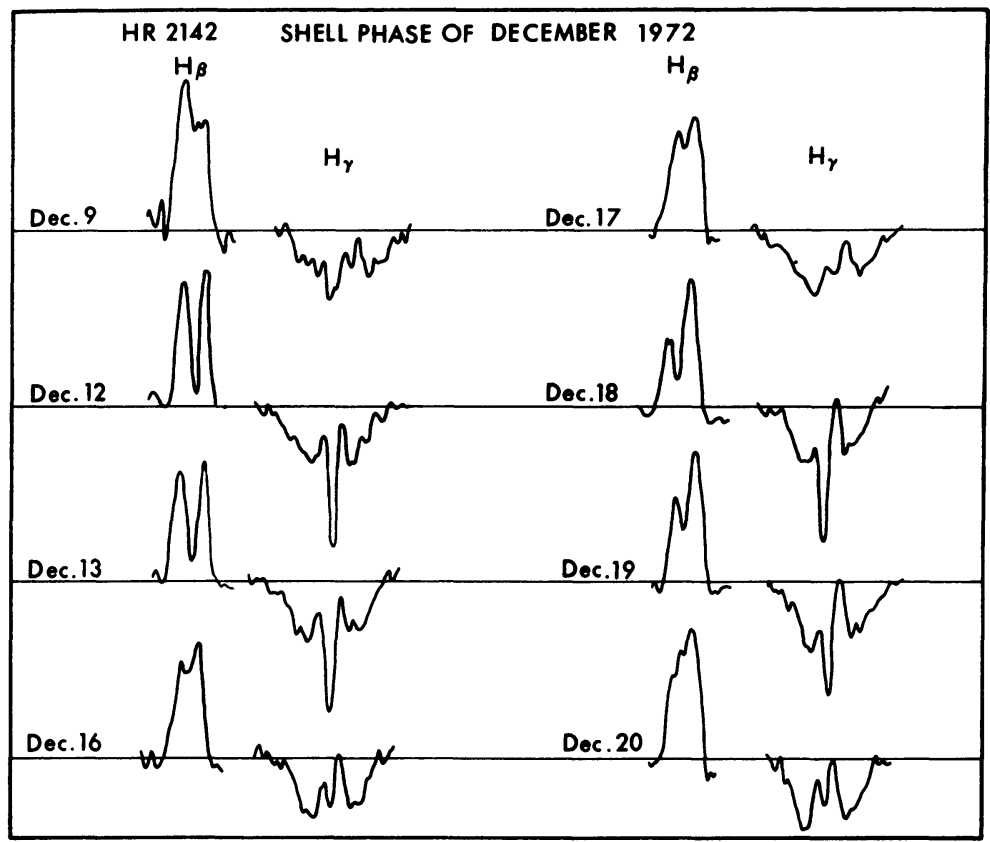

Fig. 1. The nature of the shell phase of HR 2142 is shown by this sequence of $H \beta$ and $H \gamma$ profiles observed in December 1972. The spectrograms $\left(46 \AA \mathrm{mm}^{-1}\right)$ were obtained with the UCLA 24-in. telescope at Ojai, California.

region $\lambda \lambda 4800-8700 \AA\left(11-23 \AA \mathrm{mm}^{-1}\right)$ and direct spectrograms from $\lambda \lambda 3200-$ $4900 \AA\left(11-16 \AA \mathrm{mm}^{-1}\right)$. The dispersion for the Ojai spectrograms is $46 \AA \mathrm{mm}^{-1}$.

\section{Summary of Observations to Date for HR 2142}

The sequence of events which take place during the primary shell phase of HR 2142 is described in detail by Peters (1972). In this section, the basic features of the primary shell phase are reviewed, new observations are presented, and the primary shell phase is compared with the secondary shell phase. In describing the observations, we define $\Phi_{s}=0.0$ as the midpoint of the interval of time in which the hydrogen cores are at maximum strength during the primary shell phase. The notation $\Phi_{s}$ is used in order to avoid confusion with phases based upon a binary orbit.

The two-component nature of the shell phase can easily be seen from the plot in Figure 2. As a measure of the varying hydrogen shell strength, we consider the change in the residual intensity of the $\mathrm{H} \gamma$ core. Whereas shell structure in $\mathrm{H} \beta$ can easily be seen on high dispersion plates taken five days before $\Phi_{s}=0.0$, cores do not appear in $\mathrm{H} \gamma$ until $\Phi_{s}=-2^{\mathrm{d}}$. The $\mathrm{H} \gamma$ core disappears by $\Phi_{s}=4^{\mathrm{d}}$.

The secondary shell phase comes on abruptly at $\Phi_{s}=5.5$ and lasts for only $1^{\mathrm{d}} .5$. Although we have been unable to make a complete sequence of observations during the rise to maximum strength, our observations accumulated during the past three 


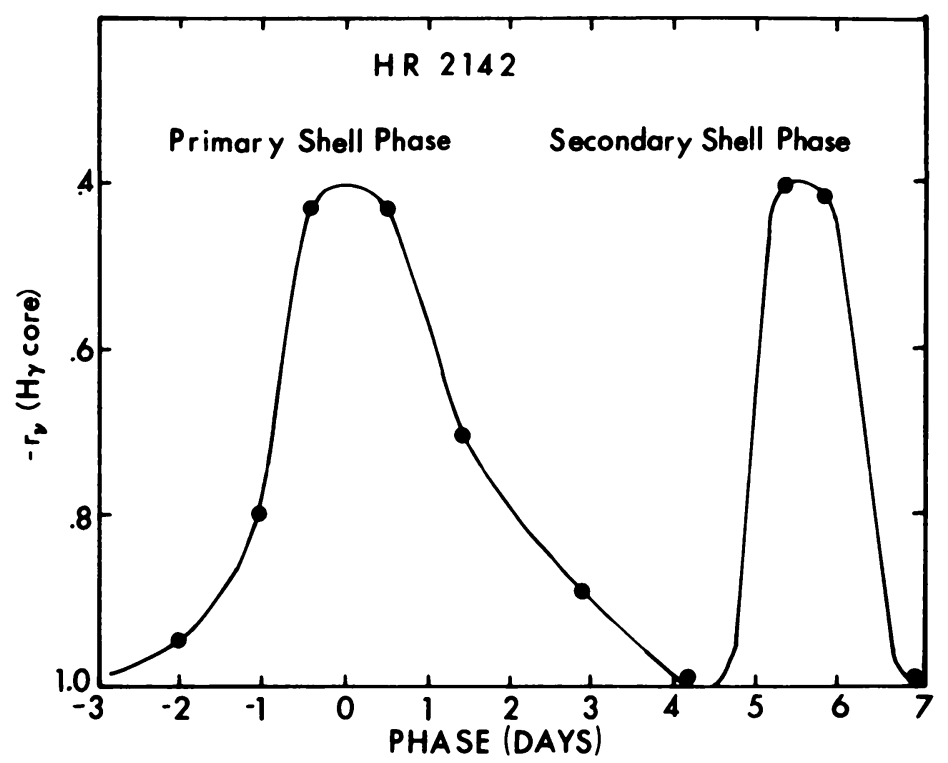

Fig. 2. The strength of the $\mathrm{H} \gamma$ core versus phase. Note the striking two-component nature of the shell phase. The data are from Lick Observatory spectrograms.

years suggest that the Balmer cores increase from zero to the same strength which they displayed during the primary shell phase in about $6 \mathrm{~h} . \lambda \lambda 5016$ and $3965 \AA$ of $\mathrm{He}$ I (which arise from the metastable $2^{1} S$ level) are seen as shell features during the secondary shell phase but not during the primary shell phase. These He I cores are -0 d 5 out of phase (they appear earlier) with the $\mathrm{H}$ cores. Although the strengths of the Balmer shell lines are comparable during both shell phases, they appear to be sharper during the secondary shell phase. The profiles of the $\mathrm{H}$ cores are slightly broader than the instrumental ones during the primary shell phase. Typically, we observe cores to $\mathrm{H} 14$ in the primary shell phase and to H20 in the secondary shell phase.

The contrast between the radial velocities exhibited by the Balmer cores from primary to secondary shell phase is as striking as the difference in their duration. The observed effect is seen in Figure 3. The Balmer shell features $(\mathrm{H} \beta$ and higher) are red-shifted relative to the photospheric features during the primary shell phase and show a steady decrease in velocity toward the photospheric value as the shell phase progresses. The $\mathrm{H} \alpha$ core is also red-shifted but appears to remain more constant in velocity throughout the primary shell phase. When the Balmer cores re-appear during the secondary shell phase, they are blue-shifted relative to the photosphere and remain constant in velocity for the entire short duration of this segment of the shell phase. A Balmer progression is observed which indicates deceleration of material along our line of sight during the primary shell phase and the reverse for the secondary shell phase.

The profile of $\mathrm{H \alpha}$ in HR 2142 varies in an interesting fashion during the shell phase. Some representative $\mathrm{H} \alpha$ profiles observed at various phases are presented in 


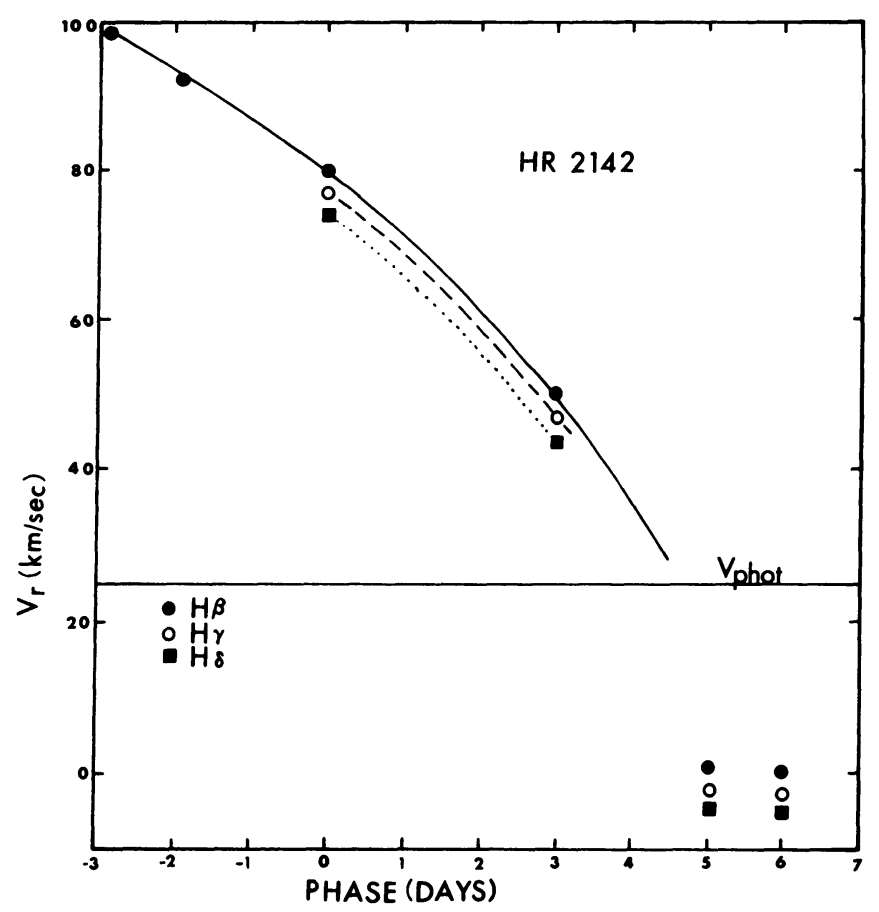

Fig. 3. The radial velocities of the Balmer shell lines as a function of phase. The velocity of the photospheric features is indicated. Note the Balmer progression.

Figure 4. Outside of shell phase, $\mathrm{H} \alpha$ is a strong emission feature $\left(I \approx 4 I_{c}\right)$ which shows no conspicuous structure. The intensity of the redward side of the line is greater than that of the blueward side, however. During the primary shell phase, we observe a strong, red-shifted core in $\mathrm{H} \alpha$ plus a weak blue-shifted $\left(\approx 150 \mathrm{~km} \mathrm{~s}^{-1}\right)$ satellite feature which appears near $\Phi_{s}=0.0$, reaches maximum strength at $\Phi_{s}=4^{\mathrm{d}}$, and remains fixed in velocity. However, the most interesting $\mathrm{H} \alpha$ profiles in HR 2142 are observed during the secondary shell phase and shortly thereafter. When deep shell lines are present in $\mathbf{H} \boldsymbol{\beta}$ and the higher Balmer features, multiple, weak cores are observed in $\mathrm{H} \alpha$. The material in the outer portion of the 'envelope structure' through which we are viewing during the secondary shell phase appears to have a wider range of velocities than the material in the deeper sections. A minimum of three components nearly equally spaced across the emission profile can always be seen during the secondary shell phase. The fine structure is as repetitive from one cycle to the next as the appearance of the deep shell absorptions in the other Balmer members. For a few days after the secondary shell phase, when shell structure has disappeared in the higher Balmer members, $\mathrm{H} \alpha$ continues to show weak structure. One noteworthy component is blue-shifted by nearly $150 \mathrm{~km} \mathrm{~s}^{-1}$ relative to the center of the emission feature.

Recent observations of $\mathrm{H} \beta$ during the post shell phase have revealed the presence of multiple weak, blue-shifted absorption cores. We feel that these absorption components are responsible for the low intensity of the $V$ lobe of $\mathrm{H} \beta$ (and the other 

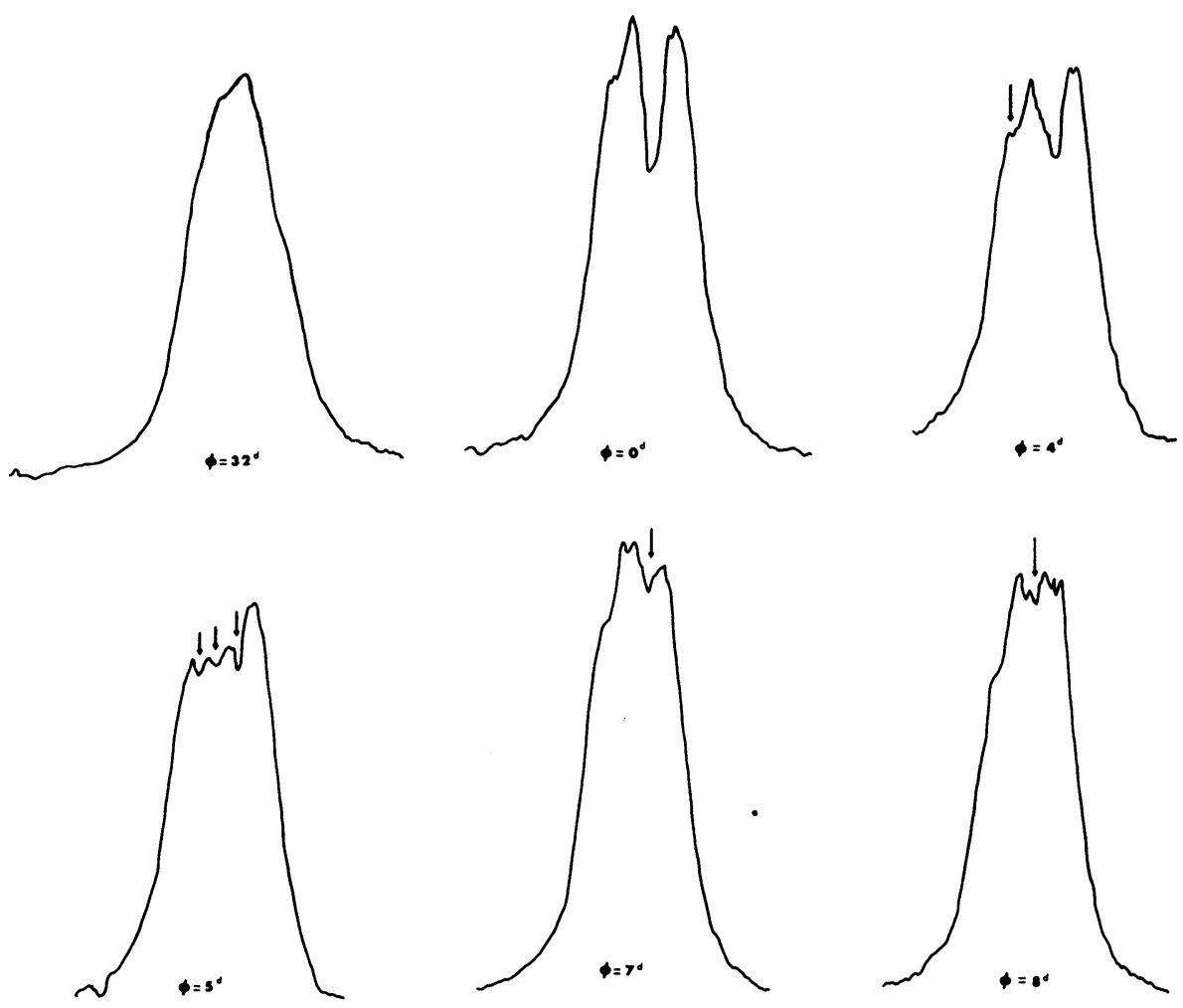

$\Delta \lambda \cdot 10 A$

He DENSITY PROFILES FOR VARIOUS PHASES

Fig. 4. Representative profiles of $\mathrm{H} \alpha$ in HR 2142 observed at selected phases. The arrows point to confirmed weak absorption components in the emission line profiles.

Balmer features) which has already been reported at this phase (Peters, 1972). The equivalent width of the missing $V$ lobe of $\mathrm{H} \beta$ is comparable to that of the $\mathrm{H} \beta$ core during the secondary shell phase.

It has already been established that the $H \beta$ profile varies in a regular manner with phase (Peters, 1972). Typical $\mathbf{H} \boldsymbol{\beta}$ profiles for various phases were presented in Figure 3 of the latter paper. Within the past three years we have gathered additional data which not only confirm the earlier result but also show that the $R$ lobe remains essentially constant in intensity throughout the cycle. The cyclic $V / R$ variations are a result of the changing intensity of the $V$ lobe. The data accumulated on $V / R$ for $H \beta$ as a function of phase appear in Figure 5. It can be seen that $R>V$ at all phases except near $\Phi_{s}=0$ ?.9.

In concluding this section of the paper, I would like to re-emphasize the strict periodicity of the shell phases and other cyclic spectral variations which are observed in HR 2142. Even fine structure in $\mathrm{H} \alpha$ repeats from one cycle to the next. The data which we have accumulated on HR 2142 in the past six years combined with Dr D. B. 


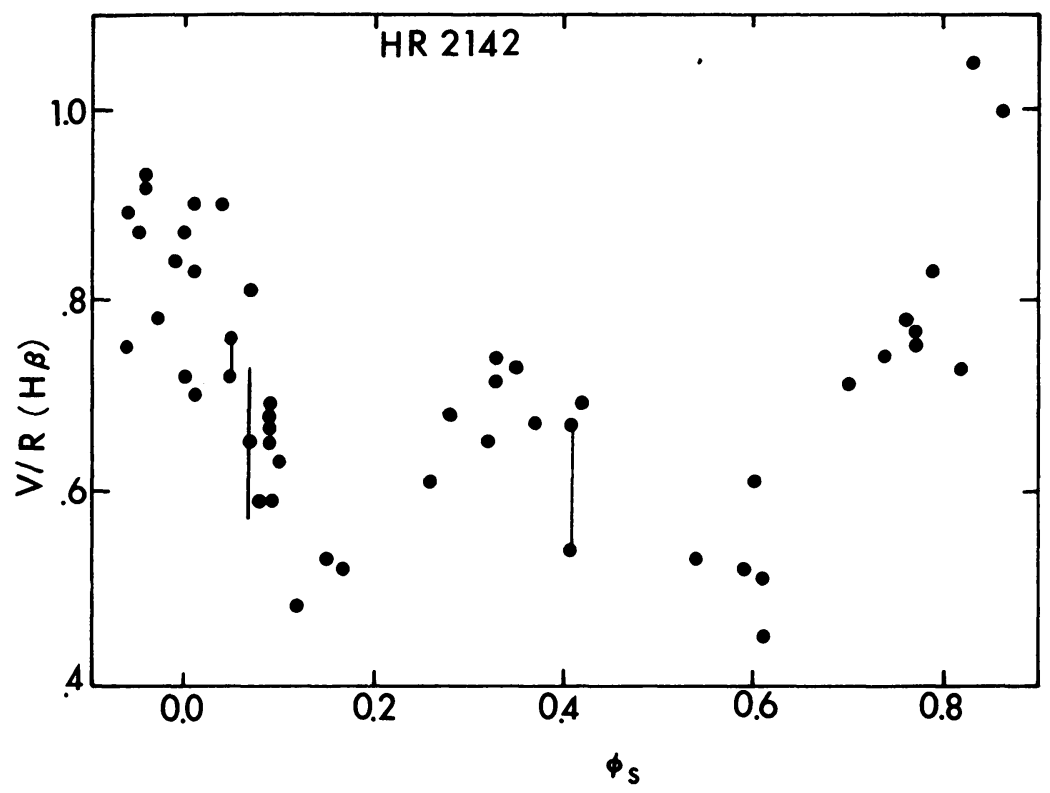

Fig. 5. The change in the ratio $V / R$ for $\mathrm{H} \beta$ with phase. Vertical lines joining two points indicate that the observations were made on the same night. The point plus vertical line at $\Phi_{s}=0.07$ represents the mean of seven observations made during one night; the length of the line indicates twice the standard deviation.

McLaughlin's spectrograms which exist in the plate files of the University of Michigan allow a refinement to the period quoted in Peters (1972). A Michigan spectrogram taken on October 6,1933 which shows conspicuous shell structure at $\mathrm{H} \gamma$ was particularly valuable in this endeavor. All available observations of HR 2142 can be fit well with $P=80^{\mathrm{d}} .860 \pm 0.015$ and $\Phi_{s}=0^{\mathrm{P}} .0$ on JD 2440855.5.

\section{A Model for HR 2142}

The strict periodicity of the two-component shell phase and the complex spectral variations observed not only during the shell phase but throughout the cycle suggest that HR 2142 is an interacting binary undergoing Case B mass exchange. The primary and secondary shell phases occur when we view the light of the B1 star through, respectively, the main gas stream and the counter streaming material. This model differs slightly from the one presented at IAU Symposium No. 51 (Peters and Polidan, 1973) in that we now feel that the material surrounding the primary extends out to $100 R_{\odot}$ (about $15 R_{*}$ ), the limit of the Roche lobe of the primary, and that there is some streaming of material near $\Phi_{s}=0.2$ and at $L_{3}$. The computations of Lubow and Shu (1975) for mass streaming were considered in constructing the dimensions of the primary stream.

A polar view of the model for HR 2142 is shown in Figure 6. The mass losing secondary has a radius of approximately $40 R_{\odot}$ and is positioned $20^{\circ}$ outside our line of sight. A single arrow shows the direction of mass flow in the primary gas stream 


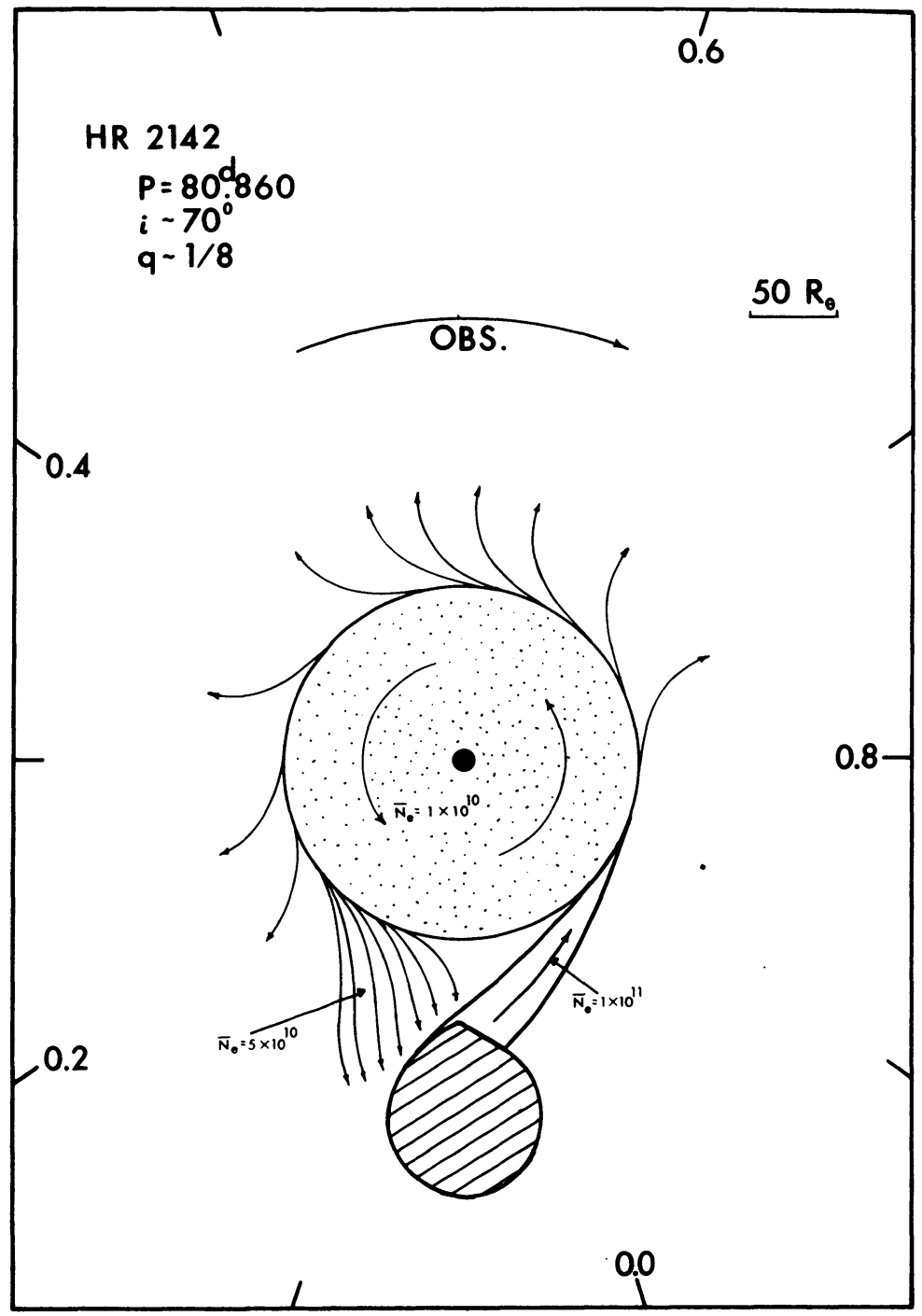

Fig. 6. A polar view of our model for HR 2142 drawn to scale. The notation and the derivation of the physical parameters are discussed in the text.

while a series of additional arrows, whose spacing are an indication of the extent of mass streaming, show other regions of mass loss. Some physical parameters obtained for the system are also noted in Figure 6. Nearly all of the observed features of HR 2142 can be explained, at least semi-quantitatively, by the model. Some of the more important and fundamental ones are discussed below.

The mass ratio which was used in the construction of the model, $1: 8$, is an assumed value. Since the primary is a B1 star, its mass must be near $12 M_{\odot}$. A mass of $1.5 M_{\odot}$ is then implied for the secondary. Since the spectrum of the secondary cannot be seen in the near infrared, its spectral type is most likely $\mathrm{G}$ to early $\mathrm{K}$ (see paper by Polidan 
presented in this session, p. 401). At the end of this section the plausibility of the assumed mass ratio will be discussed in light of some recent radial velocity measurements for HR 2142.

The fact that we do not observe eclipses suggests that the inclination of the system is less than $75^{\circ}$. However, the system must be viewed nearly edge-on since, according to our model, we are looking through a well defined stream of material. In addition, the photospheric features in HR 2142 are amongst the broadest seen in early B type stars and suggest that $v \sin i \approx 350 \mathrm{~km} \mathrm{~s}^{-1}$. Therefore, we suggest that HR 2142 is viewed nearly equator-on and that it just barely misses being eclipsed. $70^{\circ}$ appears to be a reasonable value for the inclination of the system.

The observed velocity sequence displayed by the Balmer cores during the primary shell phase indicates that the main gas stream is fairly curved and moderately focused toward the direction of the primary. At the beginning of the shell phase, when the cores are weak, we are viewing through material which has a fairly high component of velocity toward the primary $\left(\approx 100 \mathrm{~km} \mathrm{~s}^{-1}\right)$; when $\Phi_{s}=0.04$, we are looking through the stream as it is moving tangentially to our line of sight. At $\Phi_{s}=0$. 0 , when $N_{0,2} h$ is a maximum, we view the stream through some intermediate orientation. Since our line of sight intercepts the stream for one-tenth of the orbit, a lower limit on the length of the stream is $100 R_{\odot}$. Recall that the observed Balmer progression suggests a deceleration of material toward the primary.

The counter streaming material through which we are viewing during the secondary shell phase must, in the vicinity of the secondary star, have a very small velocity dispersion in our line of sight. From the duration of the secondary shell phase we estimate that the projected size of this 'focused' portion of the counter stream is $25 R_{\odot}$. The outward velocity of the counter streaming material, relative to the photosphere of the primary, should be close to the observed projected value of $25 \mathrm{~km} \mathrm{~s}^{-1}$. The blue-shifted satellite core which develops and strengthens during the primary shell phase is most likely formed in the outer edges of the counter streaming structure.

The cyclic profile variations evident in $\mathrm{H} \beta$ (and other Balmer envelope features) can be understood in terms of the model. It was mentioned in the previous section that the $V / R$ variations result from a cyclic depression of the $V$ lobe. We feel that multiple weak absorptions arising from material streaming away from the disk are responsible for the $V / R$ variations. The $V$ lobe is weakest near phases $0^{p} .15$ and 0 .55. Near $\Phi_{s}=0$. 15 we are viewing the light of the primary through a portion of the counter streaming material which has a large dispersion in velocity. If one looks carefully at the sequence of events in $\mathrm{H} \beta$ commencing with the secondary shell phase, one observes the sharp blue-shifted core broaden then move blue-ward until it 'disappears' into the $V$ lobe. The fact that the equivalent width of the missing $V$ lobe observed between $0^{\mathrm{P}} .15<\Phi_{s}<0^{\mathrm{p}} .25$ is comparable to that of the core observed during the secondary shell phase suggests that we are viewing through comparable amounts of material. We interpret the depression of the $V$ lobe near $\Phi_{s}=0^{p} .55$ as evidence of material streaming off from $L_{3}$. We do not expect a well-defined stream at $L_{3}$. Between $0^{\mathrm{p}} .8<\Phi_{s}<0^{\mathrm{p}}$. 0 , where the primary stream is joining the disk, we expect to see a minimum of high velocity blobs leaving the system. Our observations support this prediction (see Figure 5).

The mean density of the material in the envelope around the primary star was 
determined from the volume emission measure, $N_{\text {ion }} N_{e} V$, which was computed from Wellman's formula (1951; Pagel, 1960) and the measured equivalent widths of the emission features of $\mathrm{H} \beta, \mathrm{H} \gamma$, and $\mathrm{H} \delta$. If one assumes that $N_{\text {ion }}=N_{e}$ and that the envelope is a wedge-shaped disk of radius $100 R_{\odot}$ with inner and outer heights of $5 R_{\odot}$ and $25 R_{\odot}$, respectively $\left(V=10^{38} \mathrm{~cm}^{3}\right)$, then one obtains a mean electron density of about $10^{10} \mathrm{~cm}^{-3}$ for the material in the circumstellar envelope.

Estimates for the mean electron density in the two streams were obtained from the column density of absorbers, $N_{0,2} h$, deduced from the strengths of the hydrogen shell lines observed during the primary and secondary shell phases. The temperature of the gas was taken to be $12000 \mathrm{~K}$ and the assumed path lengths through the streams were $25 R_{\odot}$ and $60 R_{\odot}$ for the main gas stream and the counter streaming material, respectively. The mean density for the primary gas stream turned out to be ten times higher than the mean density in the circumstellar envelope; the density for the counter streaming material is somewhat lower than that of the main gas stream but higher than the mean density of the envelope.

Finally, one can estimate the rate of mass loss from the secondary star from the observed velocities and the computed density and dimensions of the primary gas stream. We obtain a mass loss rate between $10^{-7}$ and $10^{-6} M_{\odot} \mathrm{yr}^{-1}$.

The strict periodicity of the shell phases in HR 2142 has inspired this model. However, in principle, if HR 2142 is a binary, then one should be able to measure radial velocity variations in the primary and determine an orbit for the system. The fact that the photospheric features in HR 2142 are quite rotationally broadened coupled with the distortions which appear in the centers of the $\mathrm{He}$ I lines at certain phases have made it impossible to derive an adequate radial velocity curve from a Grant-type measuring machine. Recently, Dr J. B. Hutchings used the 'wide-scan' oscilloscopic comparitor ARCTURUS at the Dominion Astrophysical Observatory to measure the positions of the $\mathrm{He}$ and $\mathrm{H}$ features on five of the Lick plates which were taken at representative phases. His results are shown in Figure 7. The measurements not only produced a radial velocity curve but also indicated that conjunction occurs between the primary and secondary shell phases. Since $\mathrm{K}$ is about $20 \mathrm{~km} \mathrm{~s}^{-1}$, the mass function is about $0.07 M_{\odot}$. If $M_{p}=12 M_{\odot}$, then $M_{s}=2.5 M_{\odot}$. In

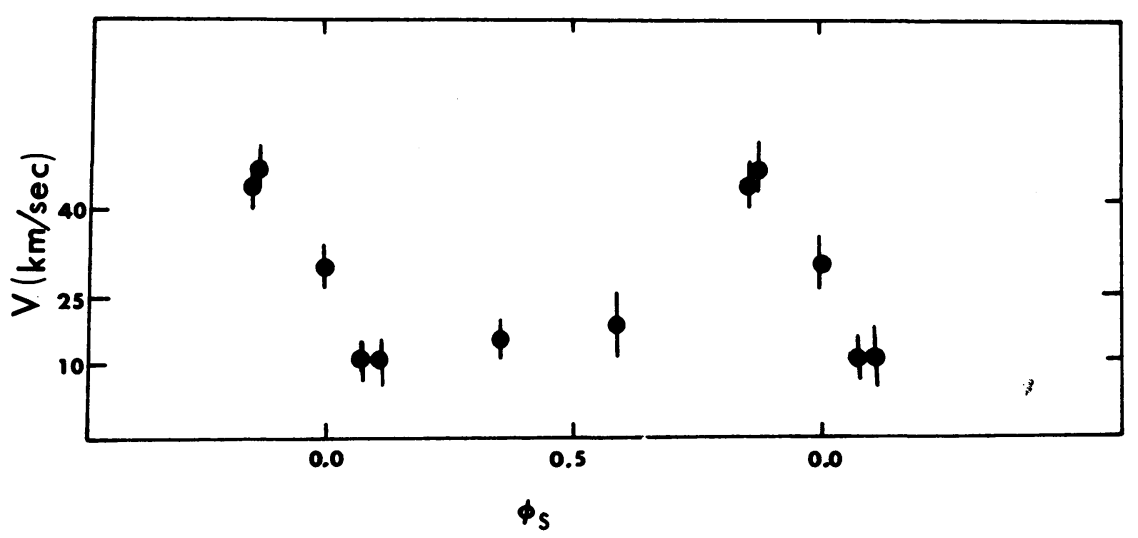

Fig. 7. The radial velocity curve for HR 2142 suggested by Hutchings' measurements. Double points indicate two measurements of the same plate. 
this case the mass ratio of the system would be closer to $1: 5$ than $1: 8$. Hutchings' measurements suggest an elliptical orbit. I feel that the orbit must be fairly circular due to the regularity of the shell phases. Velocities near $\Phi=0.5$ could be affected by gas streaming from $L_{3}$. The orbit should certainly be regarded as preliminary at this time. Additional measurements which will allow us to refine the orbit are planned in the immediate future.

\section{Comparison between HR 2142 and other Binary Be Stars}

In this section we briefly compare the spectral behavior of some confirmed $\mathrm{Be}$ binaries with HR 2142. Before we concern ourselves with the details of each object, however, it should be stated that a two-component shell phase of short duration as we observe in HR 2142 has not been observed in any other Be star. Thus, the spectral variations in HR 2142 do remain unique.

AXMon (B1e + K2II; $P=232$ d 5 ) has been studied extensively since the early 1920's (Merrill, 1923). More recently the cyclic spectral variations in this star have been investigated by Cowley (1964) and Peton (1974). AX Mon periodically develops a strong hydrogen and metallic shell six weeks before conjunction. Maximum shell strength is reached three weeks before conjunction and the velocities of the shell features are positive relative to the photosphere during the initial segment of the shell phase. The observations strongly indicate the presence of gas streaming. However, AX Mon differs from HR 2142 in that shell features are seen over most of the orbit (they are absent for only $20 \%$ of the time). In addition, HR 2142 does not show a metallic line shell in the ground based portion of its spectrum. Strong hydrogen shell lines and metallic shell features are observed $20 \%$ of the time in AX Mon; HR 2142 shows a conspicuous hydrogen shell $6 \%$ of the time.

17 Lep (B9e $+M 1 ; P=260^{d}$ ) has been studied in detail by Cowley (1967). This star tends to undergo outbursts at 30-40 days past the time of periastron passage. However, shell lines which are blue-shifted relative to the permanent shell features are observed at all phases. The fact that the orbit is eccentric $(e \approx 0.1)$ is most likely responsible for the high percentage of unpredictable shell outbursts. Unlike AX Mon and HR 2142, 17 Lep is viewed nearly pole-on.

HD $218393\left(\mathrm{~B} 2 \mathrm{e}+\mathrm{K} 1 \mathrm{II} ; \boldsymbol{P}=39^{\mathrm{d}}\right.$ ) shows periodic variations in the radial velocities and intensities of the hydrogen shell lines. Doazan and Peton (1970) have extensively studied this star and interpret the velocity variations as evidence of an oscillating envelope. Cyclic variations in the profile of $H \beta$ of the $V / R$ type are also observed. For most of the cycle, $R \gg V$. However, unlike HR 2142, both components vary in intensity. The spectrum of the secondary has recently been discovered (Polidan and Peters, 1975). At this time it is not clear how mass exchange enters into the observed picture for HD 218393.

HD 173219 (B0e; $P=58^{\mathrm{d}}$ ) shows large amplitude radial velocity variations $\left(\approx 200 \mathrm{~km} \mathrm{~s}^{-1}\right)$. Hutchings and Redman (1973) have studied this star in detail and

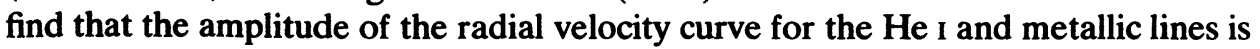
lower than the one for the $\mathrm{H}$ cores. Some of the metallic lines are variable with phase and may be partially formed in the circumstellar envelope. Hutchings and Redman 
suggest that the secondary may be a collapsed object. Incidentally, the spectrum of HD 173219 in the near infrared closely matches that of HR 2142.

$\phi \operatorname{Per}(\mathrm{B} 0 \mathrm{e})$ displays a cyclic strengthening and fading of a $\mathrm{H}$ and $\mathrm{He}$ shell with a period of 126.696 . The spectral variations in this star have been studied in detail by Hynek (1940) and Hickok (1972). The spectrum of $\phi$ Per resembles that of HR 2142 in that both objects show (1) a strong, near featureless $\mathrm{H} \alpha$ emission feature, (2) emission of Fe II, O I $\lambda 7774 \AA, O_{\text {I }} \lambda 8446 \AA$, and the infrared Ca II triplet, and (3) extensively broad photospheric features. However, the shell sequence in $\phi$ Per is quite different from that in HR 2142. Balmer shell features are observed for most of the cycle in $\phi$ Per. During one segment of the cycle, the velocities of the hydrogen cores and the $\mathrm{He}$ I shell lines which arise from metastable levels increase noticeably $\left(\Delta V \approx 40 \mathrm{~km} \mathrm{~s}^{-1}\right)$ with time, then the $\mathrm{H}$ and $\mathrm{He}$ cores abruptly disappear for $15^{\mathrm{d}}$. Subsequently, the cores remain constant in velocity for $40^{\mathrm{d}}$, then show multiple components. In addition, $\mathrm{He} \mathrm{I}$ cores appear when the $\mathrm{H}$ shell lines show constant velocity.

All of the Be binaries which have been described above, including HR 2142, show emission at the infrared $\mathrm{Ca}$ II triplet. The paradox of the $\mathrm{Ca}$ II triplet and its possible connection with binary nature were discussed by $R$. S. Polidan in the preceding paper.

\section{Conclusions and Suggestions for Future Work}

Cyclic spectral variations with periods of the order of $100^{d}$ apparently do characterize most binary Be stars. These variations can be of several types and include periodic shell activity (HR 2142, AX Mon, $\phi$ Per), cyclic variations of the emission line profiles (HR 2142, HD 218393), and subtle cyclic variations of the profiles of the photospheric features (HD 173219). Of course, one can construct a variety of models based upon the concept of binary mass transfer and, thus, predict a wide range of observed envelope spectra and shell sequences. The stage of mass transfer, the mass ratio of the system, the separation of the components of the system, and the inclination of the system to our line of sight determine what one observes. In fact, we can potentially learn a great deal about the details of binary mass transfer from studying the Be stars which are confirmed interacting binaries.

I suggest that it may prove fruitful to look for periodic shell phases in other Be stars. I refer specifically to searching for variations with periods around $100^{d}$. Certainly it is a difficult observational problem to search for periodic short-termed shell structure as one sees in HR 2142. Consider, if short-termed shells were strong and common in Be stars, they most likely would have been discovered. The periodic shell phase in HR 2142 was elusive enough, as D. B. McLaughlin observed the star forty times in thirty years and missed discovering it even though shell structure was present on $\frac{1}{5}$ of his plates. Ideally, one needs 'continuous' coverage at $\mathrm{H} \alpha$ or $\mathrm{H} \beta$ at dispersions of $16 \AA \mathrm{mm}^{-1}$ or higher. It may prove easier to look for cyclic profile variations with periods near $100^{\mathrm{d}}$. HR 2142 and other Be stars which show periodic spectral variations have shown us that we are in need of more data on the constancy (or variability) of Be stars on the time scale of $100^{d}$. 


\section{Acknowledgements}

I wish to acknowledge many interesting and helpful discussions on periodic shell activity and mass transfer with R. S. Polidan and Dr M. Plavec. The model of HR 2142 presented in this paper was formulated jointly with R. S. Polidan. Dr M. Plavec has allotted generous amounts of his time on the Lick Observatory 120-in. telescope for this project on HR 2142. For this I am very grateful. I would like to thank E. A. Harlan of Lick Observatory and M. V. Wright of UCLA for numerous supplementary plates of HR 2142. In fact, many of the observations presented in Figure 1 were made by M. V. Wright. Finally, I wish to thank Dr J. B. Hutchings for measuring the Lick Observatory spectrograms on the comparitor of the Dominion Astrophysical Observatory. This project is part of a broad investigation of the structure and evolution of close binary stars and was partially supported by NSF MPS 74-04194A01 (Popper/Plavec).

\section{References}

Doazan, V. and Peton, A.: 1970, Astron. Astrophys. 9, 245.

Cowley, A. P.: 1964, Astrophys. J. 139, 817.

Cowley, A. P.: 1967, Astrophys. J. 147, 609.

Hickok, F. R.: 1973, preprint.

Hutchings, J. B. and Redman, R. O.: Monthly Notices Roy. Astron. Soc. 163, 219.

Hynek, J. A.: 1940, Contrib. Perkins Obs. $2,1$.

Lubow, S. H. and Shu, F. H.: 1975, Astrophys. J. 198, 383.

Merrill, P. W.: 1923, Publ. Astron. Soc. Pacific 35, 303.

Pagel, B. E. J.: 1960, Vistas in Astronomy 3, 203.

Peters, G. J.: 1972, Publ. Astron. Soc. Pacific 84, 334.

Peters, G. J. and Polidan, R. S.: 1973, in A. H. Batten (ed.), 'Extended Atmospheres and Circumstellar

Matter in Spectroscopic Binary Systems', IAU Symp. 51, 174.

Polidan, R. S. and Peters, G. J.: 1975, this volume, 59.

Peton, A.: 1974, Astrophys. Space Sci. 30, 481.

Wellman, P.: 1951, Z. Astrophys. 30, 71.

\section{DISCUSSION}

Delplace: How many cycles have you observed for HR 2142?

Peters: Of the order of 20.

Polidan: Based on the old plates, it appears that HR 2142 has shown the same shell activity with the same period for roughly 50 years.

Note added in proof: In December 1975, the higher dispersion spectrograms of HR 2142 were measured with ARCTURUS at the Dominion Astrophysical Observatory. An orbit was obtained for HR 2142 which supports our model $\left(K \simeq 10 \mathrm{~km} \mathrm{~s}^{-1}, e \simeq 0\right.$, and conjunction near $\left.\Phi_{s}=0^{\text {P.05}}\right)$. Since $f(m)=0.008 M_{\odot}$, reasonable masses for the primary and secondary are $12 M_{\odot}$ and $1.2 M_{\odot}$, respectively. 